\title{
Is he the culprit?
}

\section{Pradhan BB, Shrestha S}

Department of Medicine

Narayani Sub-regional Hospital, Birgunj

\section{ABSTRACT}

The peripartum cardiomyopathy (PPCM) generally occurs in the last trimester of pregnancy or within 6 months of delivery. The disease generally occurs in multiparous female more than 30 years of age. The prognosis depends upon whether the heart returns to normal size after the first episode of CHF. If heart remains enlarged or the ejection fraction remains depressed after 6 months, the prognosis is poor ${ }^{1}$.

We present a case of 27 years female who gave birth to her fourth child and presented peripartum cardiomyopathy 11 months after the child birth. She was admitted, evaluated and managed with diuretics in the initial period and when stabilized placed on ACE inhibitor and alpha beta blocker. She improved significantly and was discharged. She was on regular follow up and showed a significant improvement in heart size, ejection fraction and valvular function when re-evaluated after 7 months.

\section{Key wods}

Peripartum cardiomyopathy, echocardiography, congestive cardiac failure 


\section{Introduction}

Peripartum cardiomyopathy (PPCM) is an idiopathic cardiomyopathy that presents with heart failure secondary to left ventricular systolic dysfunction toward the end of pregnancy or in the months after delivery, in the absence of any other cause of heart failure. PPCM is a diagnosis of exclusion. Although the left ventricle may not be dilated, the ejection fraction is nearly always reduced below $45 \%$. This is the definition adopted by Heart Failure Association of the European Society of Cardiology Working Group on PPCM 20103. Multiparity, black race, elderly gravida and gestational hypertension are noted risk factor but the exact cause is unknown though nutritional, viral, genetic and immune mechanism has been hypothesized contributing to $\mathrm{PPCM}^{4}$. The incidence of PPCM has a wide variation among countries ranging from 1 per 1000 live birth to 1 per 6000 live birth ${ }^{4}$.

\section{Case Report}

Here we are reporting a case of 27 years multiparous, female $\left(\mathrm{P}_{4+3}\right)$ from Rautahat district who had given birth to a live baby 11 months back, normal vaginal delivery at home, presented to emergency department with complains of easy fatiguability for last 7 days. She also complained of palpitation and gradually worsening shortness of breath since then. Initially she had shortness of breath on extreme exertion but from last 2 days she got breathless even with minimal exertion. She said her symptoms worsen when she lie down than when she sat upright. However there was no history of fever, cough and chestpain. She was nondiabetic, nonhypertensive. She never smoke or drank alcohol. There was no past history of any heart disease and she never had such symptoms on her previous pregnancies and child birth. On examination, she was a bit restless with tachycardia, pulse rate 130 per minute and tachypnea with respiratory rate 28 per minute. Her blood pressure was $100 \mathrm{mmhg}$ systolic and $60 \mathrm{mmhg}$ diastolic. She was not febrile. There was no icterus, no pallor and no pedal edema. On auscultation she had pulmonary rales and basal crepitations spreading upto half of lower chest bilateral. Cardiac auscultation revealed a systolic murmer over the mitral area. She was advised for a Chest X-ray, ECG and Echocardiography. Chest $X$ ray PA view was typical of pulmonary edema with scattered patchy opacities on lower zone and cardiomegaly. The ECG showed sinus tachycardia, first degree AV block and nonspecific ST and T segment changes. Echo revealed marked LV systolic dysfunction, EF 25-30 \%, moderate MR and LVID 61.7mm diastole. A provisional diagnosis of congestive cardiac failure was made. The patient was admitted and managed with fluid and salt restriction, diuretics, antibiotics and proton pump

inhibitors. She gradually showed improvement. The ace inhibitor was added on 3rd day admission along with alpha beta blocker. The dose of alpha beta blocker was titrated. She was discharged on fifth day with diuretics, a combination of spironolactone $50 \mathrm{mg}$ and furosemide 40 $\mathrm{mg}$ half tablet at 8am once daily, enalapril $2.5 \mathrm{mg}$ once a day and carvedilol $3.125 \mathrm{mg}$ twice daily and was advised to follow up in 2 week. Her hospital stay was uneventful and at the time of discharge her blood pressure was 110/70 $\mathrm{mm} \mathrm{hg}$, pulse 90 per minute, respiratory rate 20 per minute and temperature $98^{\circ} \mathrm{f}$. The chest was clear on auscultation but the systolic murmur still audible at mitral area. She paid regular visit to the OPD. An echocardiography repeated at 7 month showed a significant improvement in ejection fraction $40-45 \%$, MR was also reduced and LVID improved $57.9 \mathrm{~mm}$ diastole.

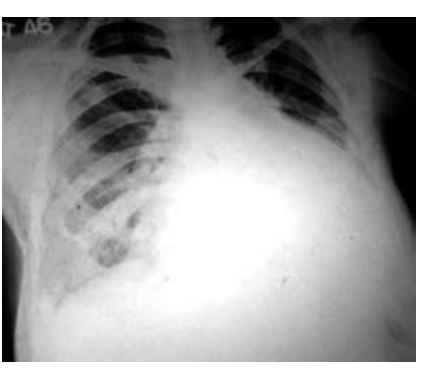

FIG 1 chest xray at the time of admisson

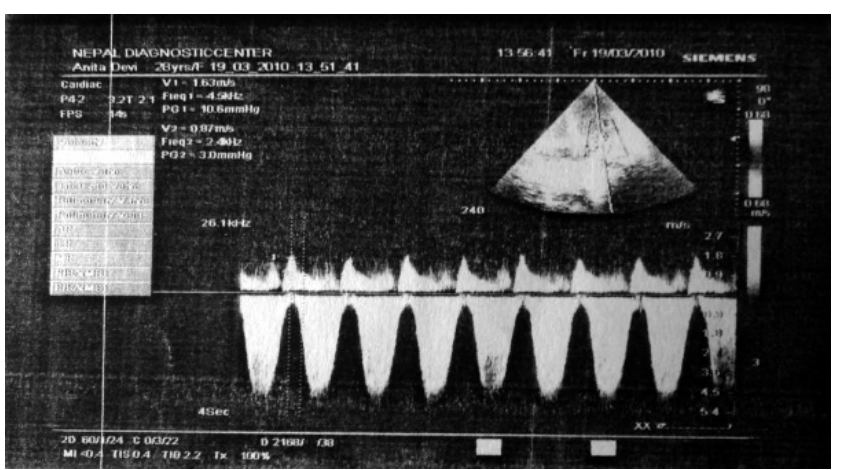

FIG 3. Echocardioggraphy at the time of admisson

\section{Discussion}

The first case of PPCM was reported in $1800 \mathrm{~s}^{4}$. The entity is associated with every 1000 to 6000 pregnancy in the world ${ }^{4}$. There have been no studies about its incidence in Nepal. The diagnostic criteria includes 1) development of cardiac failure in last month of pregnancy, 2) absence of any identifiable cause for heart failure 3) absence of recognizable heart disease before last month of pregnancy and 4) left ventricular dysfunction (EF less than 45\%). The etiology is not clear. The nutritional factor especially deficiency of selenium, viral myocarditis, genetic factors and immune mechanism has been implicated. Risk factor includes multiparity, black race, older maternal age and 
gestational hypertension ${ }^{3}$. The disease presents with sign and symptoms of congestive cardiac failure which overlap with many other condition ranging from normal pregnancy to pulmonary emboli and upper respiratory tract infection. Laboratory investigations including cardiac enzymes and pre-eclampsia work up should be considered but there are no specific laboratory abnormalities. Imaging studies include chest radiography, electrocardiogram and two dimensional echocardiography. ECG may show sinus tachycardia, nonspecific ST ant $\mathrm{T}$ wave abnormalities and voltage abnormalities. Patchy infiltrates in the lower lung fields, with vascular redistribution/cephalization, cardiomegaly and pleural effusions indicate congestive heart failure and may be seen in chest radiography. Echocardiography should be performed in all women in whom the diagnosis of PPCM is considered in order to assess ventricular function, valve structure, chamber size, and wall motion. The treatment is similar to other forms of CCF i.e. fluid and salt restriction, diuretics and beta blockers. The ACE inhibitors are contraindicated in pregnancy and the use of digoxin, nitrate and hydralazine should be considered. PPCM is associated with a high rate of thromboembolic complications. Cases of arterial or venous thrombosis have been reported in as many as $50 \%$ of women with PPCM. Because pregnancy is a hypercoagulable state, once the diagnosis of PPCM is established, prophylactic anticoagulation should be considered during pregnancy ${ }^{3}$. The delivery of the baby should be planned with a consultant gynecologist. Prognosis seems dependent on recovery of left ventricular function. $30 \%$ percent of patients return to baseline ventricular function within 6 months, and $50 \%$ of patients have significant improvement in symptoms and ventricular function. Mortality is directly related to recovery of ejection fraction ranging from $7-50 \%$ half death occurring within 3 months of pregnancy. The usual causes of death are progressive, heart failure, arrhythmia, or thromboembolism. Women with persistently abnormal ejection fractions are at high risk of developing heart failure and worsening cardiac function if they become pregnant again. A patient has so much expectation out of her pregnancy and child birth and when such incidence comes along, they get so much helpless and depressed. So a physician should provide support to the patient and discuss all the issues with her and her family members. He should assure her that this was not due to anything that she do or didn't do. A physician should always keep this in mind while working out a dyspneic female patient around her pregnancy or child birth.

\section{References}

1. Brawnwald, Fauci, Harrisson Principle of Internal Medicine $17^{\text {th }}$ edition

2. Lippy $\mathrm{p}$, Bonow RO, Braunwalds Heart Disease, a Text Book of Cardiovascular Medicine $8^{\text {th }}$ edition

3. Pregnancy and cardiomyopathy, emedicine.medscape.com/ article/153153

4. Peripartum cardiomyopathy.pdf. docsgoogle.com/viewer

5. Journal of American college of cardiology sciencedirect.com/ science/article/pii/S073510978680091 